\title{
Dissipative Rogue Waves Generated by Chaotic Pulse Bunching in a Mode-Locked Laser
}

\author{
C. Lecaplain, ${ }^{1}$ Ph. Grelu, ${ }^{1}$ J. M. Soto-Crespo, ${ }^{2}$ and N. Akhmediev ${ }^{3}$ \\ ${ }^{1}$ Laboratoire Interdisciplinaire Carnot de Bourgogne, U.M.R. 6303 C.N.R.S., Université de Bourgogne, \\ 9 avenue A. Savary, BP 47870 Dijon Cedex 21078, France \\ ${ }^{2}$ Instituto de Óptica, C.S.I.C., Serrano 121, 28006 Madrid, Spain \\ ${ }^{3}$ Optical Sciences Group, Research School of Physics and Engineering, The Australian National University, \\ Canberra ACT 0200, Australia
}

(Received 14 December 2011; published 5 June 2012)

\begin{abstract}
Rare events of extremely high optical intensity are experimentally recorded at the output of a modelocked fiber laser that operates in a strongly dissipative regime of chaotic multiple-pulse generation. The probability distribution of these intensity fluctuations, which highly depend on the cavity parameters, features a long-tailed distribution. Recorded intensity fluctuations result from the ceaseless relative motion and nonlinear interaction of pulses within a temporally localized multisoliton phase.
\end{abstract}

DOI: 10.1103/PhysRevLett.108.233901

PACS numbers: 42.65.Tg, 47.20.Ky

Waves of extreme amplitude, or "rogue waves," have been the subject of intense research activity during the past decade $[1,2]$. Initiated first in the context of oceanic waves, where the induced stakes and consequences are the most critical, the notion of "rogue waves" or "freak waves" is presently being transferred to the solid grounds of the research laboratories [3]. Observations and predictions of rogue wave events are ubiquitous in various scientific fields, from capillary waves [4] and optics [5] to plasma waves [6]. In particular, optics offers a great advantage related to the significantly faster field evolution and to the higher number of events that can be recorded in a reasonable amount of time, in contrast to research prospects in oceanography. Optical rogue waves, since their observation in the experiments with supercontinuum generation [5], have stimulated research in diverse areas of nonlinear optics. Multiple soliton collisions [7], as well as interaction with dispersive waves [8], have been identified as the key mechanisms in the formation of giant solitons, but direct experimental evidence of these mechanisms has remained elusive.

Although optical rogue waves have been mostly studied in conservative systems $[9,10]$, recent reports have placed a special emphasis on the role of active media and dissipative systems to drive instabilities to the level of rogue wave formation. These systems comprise nonlinear driven cavities [11], Raman fiber amplifiers and lasers [12,13], pump-modulated fiber lasers [14], and optically injected semiconductor lasers [15]. Large fluctuations of the energy of the output pulse were reported in a Ti:Sapphire modelocked laser operating in the single-pulse regime [16]. We here demonstrate the existence of a novel mechanism of rogue wave formation, which is based on nonlinear pulse interaction in a multiple-pulse mode-locked laser.

Indeed, two independent theoretical works recently predicted that mode-locked lasers operating in a chaotic multipulse regime might generate high-intensity optical peaks satisfying the criteria of rogue waves $[17,18]$. In order to strongly interact, the multiplicity of pulses should be confined within a relatively tight temporal domain. The two predictions also stressed the dominant impact of dissipative effects on the involved nonlinear dynamics. Dubbed "dissipative rogue waves" (DRW) [17], the high-intensity transient pulse formations result from the nonlinear interaction of several pulses having chaotic relative motions, while the bunch of pulses propagates round the laser cavity. This dynamics is fundamentally different from the fluctuations of the total cavity energy involved in the single pulse case [16]. In the present Letter, we report the first experimental observation of extreme peak-optical-intensity events created through the novel multipulse interaction mechanism described above.

Mode-locked lasers, besides being attractive sources of ultrashort optical pulses for many applications, constitute an ideal platform for the fundamental exploration of complex dissipative nonlinear dynamics [19]. Mode-locked operation with multiple pulses per cavity roundtrip is favored, in a fiber laser cavity, by the conjunction of anomalous dispersion and Watt-level pumping power. The coexisting pulses, or dissipative solitons, strongly interact. Even primarily weak interaction processes are amplified due to the endless recirculation of pulses in the cavity. Interaction of pulses through their tails, or through dispersive waves, can group all dissipative solitons into a bunch much shorter than the cavity round-trip time [17,20]. Since interactions are strongly affected by the cavity parameters, varying these parameters allows us to explore various collective pulse behaviors.

In this work, we use a fiber ring laser mode-locked through nonlinear polarization evolution (NPE). NPE, when followed by intensity discrimination in a polarization splitter, provides an ultrafast effective saturable absorption of light. The setup is shown in Fig. 1(a). The all-fiber ring laser cavity has dual 980-nm pumping of a 2-m-long 


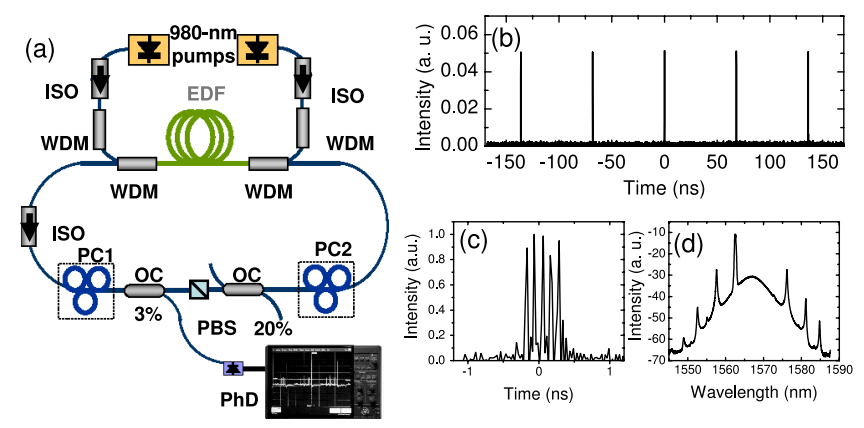

FIG. 1 (color online). (a) Fiber laser experimental setup. (b) Temporal trace of a stationary bunch of pulses circulating at the fundamental cavity repetition rate of $17.2 \mathrm{MHz}$. (c) Temporal magnification of the $\sim 1$-ns-long bunch using a $20-\mathrm{GHz}$ oscilloscope with a $45-\mathrm{GHz}$ photodiode. (d) Optical spectrum (log scale).

erbium-doped fiber (EDF, normal dispersion $D=-12.5 \mathrm{ps} \cdot \mathrm{nm}^{-1} \cdot \mathrm{km}^{-1}$ ). The maximum injected power is $800 \mathrm{~mW}$. The ring includes a polarizationinsensitive optical isolator (ISO) that ensures unidirectional laser emission at $\lambda \sim 1.5 \mu \mathrm{m}$. It also has a polarization controller made of small fiber loops (PC1), a $3 \%$ output coupler (OC), a polarization beam splitter (PBS), a four-port 80:20 coupler, and a second polarization controller (PC2). Except for a short length $(0.15 \mathrm{~m})$ of birefringent fiber at the output of the polarization splitter, components are pigtailed with SMF-28 (anomalous dispersion $D=+16.5 \mathrm{ps} \cdot \mathrm{nm}^{-1} \cdot \mathrm{km}^{-1}$ ). The overall cavity length is 12 meters, yielding a roundtrip time of $58 \mathrm{~ns}$. The cavity operates at an anomalous path-averaged chromatic dispersion $\left(D=+10 \mathrm{ps} \cdot \mathrm{nm}^{-1} \cdot \mathrm{km}^{-1}\right)$.

Mode-locking using NPE offers us many degrees of freedom to shape the transfer function of the effective saturable absorber by precisely adjusting the orientation of the polarization controllers. Such shaping drastically affects the balance between dissipative effects, as well as the amount of quasicontinuous waves that can coexist with soliton pulses in the cavity [20]. Although the nonlinear transfer function cannot be easily deduced from a particular set of angles, we can, nevertheless, explore these regimes in experiments in a perfectly reproducible way. This enables the observation of various collective pulse dynamics, with bunches comprising typically 5 to 50 pulses. The laser output is sent to a $45-\mathrm{GHz}$ photodiode, whose electrical signal is recorded by a $20-\mathrm{GHz}, 40-\mathrm{GSa} / \mathrm{s}$ real-time oscilloscope (LeCroy WaveMaster 820Zi-A) that has a rise time of 22 ps. The temporal resolution is thus at least an order of magnitude shorter than the typical pulse bunch duration of $\sim 1 \mathrm{~ns}$, allowing real-time analysis of the internal bunch structure, as illustrated in Fig. 1(c). The selected regime here corresponds to a 5-soliton bunch that travels the cavity in a stationary way. It is obtained at a pumping power of $200 \mathrm{~mW}$. The optical spectrum of Fig. 1(d) is typical of fiber laser soliton operation [20].
Varying the cavity parameters from this basic mode of operation, we observe several bifurcations, where the stability and the number of pulses are drastically affected. The increase of the pumping power to $600 \mathrm{~mW}$ augments the number of pulses as well as the trend to bunch destabilization. Once this level of pumping power is selected, the degrees of freedom bound to the polarization controllers allow for the exploration of neighboring dynamical regimes as well as bifurcations to chaotic regimes. As several laser parameters can be varied, the route to a chaotic state can also vary. Either a sequence of period doubling bifurcations [17] or a direct transition to chaos [18] can be observed.

An example of chaotic bunch dynamics is illustrated by Fig. 2. The aggregated pulses no longer occupy stationary relative positions. Their locations fluctuate randomly, producing a chaotic optical intensity distribution. In this regime, the optical spectrum, shown in Fig. 2(b), differs significantly from the soliton spectrum presented in Fig. 1(d). The strong quasi-cw sidebands are absent, and the spectrum acquires a double-hump profile with a narrow rms spectral width of around 5-nm. There are various chaotic states in laser dynamics. "Noise-like" pulse operation is one of them, and it is always characterized by an ultrawide optical spectrum [21], in contrast to the relatively narrow spectra recorded here.

Pulse clusters shown in Fig. 2(a) appear also in the numerical simulations of the laser operation (see Fig. 4 of [17]). The combination of chaotic dynamics, strong nonlinear interactions, and pulse collisions produces events of extreme amplitude and short duration. Although the actual intensity distribution is convoluted by the 22-ps response time of the fast acquisition electronics, we are able to capture internal pulse dynamics with high resolution. An example of statistical analysis
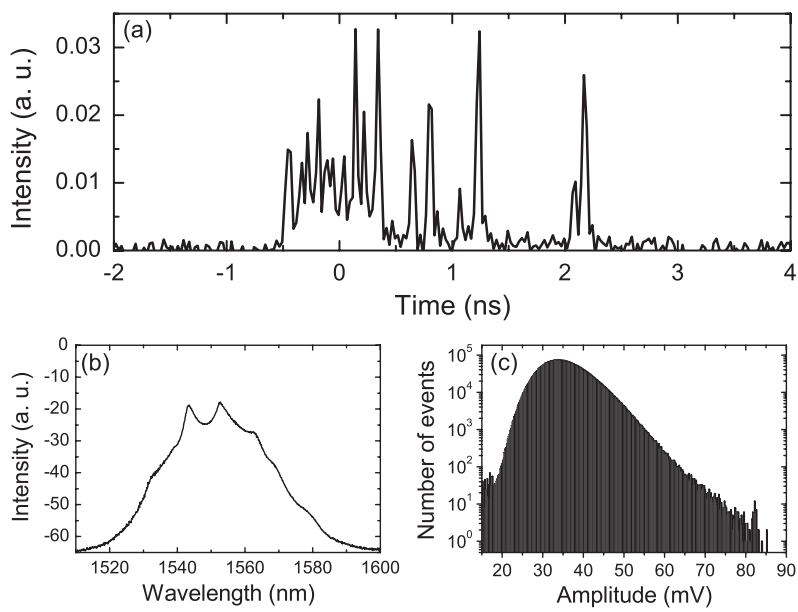

FIG. 2. (a) Optical intensity record of a chaotic bunch of pulses (b) Optical spectrum (log scale) (c) Histogram (log scale) showing the distribution of the optical intensity maxima for $4.9 \times 10^{6}$ trace events. SWH is $41.9 \mathrm{mV}$ 
illustrating the occurrence of large pulse intensity fluctuations is displayed on Fig. 2(c). The recording process is as follows. The laser output is attenuated to avoid saturation in the fast detector. The latter converts linearly the optical intensity into an electrical voltage $(\mathrm{mV})$. The $20-\mathrm{GHz}$ realtime oscilloscope records one trace representing the intensity evolution inside one roundtrip, and measures the peak intensity within this record. The whole process is automatically repeated at a rate of $\sim 10^{6}$ trace samples per hour with the peak intensity records being added to a histogram. We can see that the long-tailed histogram shown in Fig. 2(c) displays numerous large intensity events, thus revealing the generation of rogue waves (RW) in the cavity.

Three main features define a RW phenomenon [3]. First, the dynamics should exhibit rare wave events of giant amplitude. This is determined by comparison of a given wave amplitude to the significant wave height (SWH), defined as the mean amplitude of the highest third of the waves. Wave events of amplitude larger than twice the $\mathrm{SWH}$, are extreme waves that qualify. This criterion is generally sufficient to distinguish RWs from regular pulsations that abound in dissipative nonlinear dynamics [22] and from chaotic pulsations such as dissipative soliton explosions [23]. To be termed as RWs, they should also be transient waves that appear unexpectedly and disappear without a trace. This second criterion excludes tsunamis, for instance, whose propagation can be traced over long distances. The third criterion states that RW events arise more frequently than they should if they were obeying a classical Rayleigh distribution or Gaussian statistics. These three criteria are satisfied in the case of the chaotic pulse bunch presented in Fig. 2. In particular, the high-amplitude part of the histogram reveals extreme events with larger

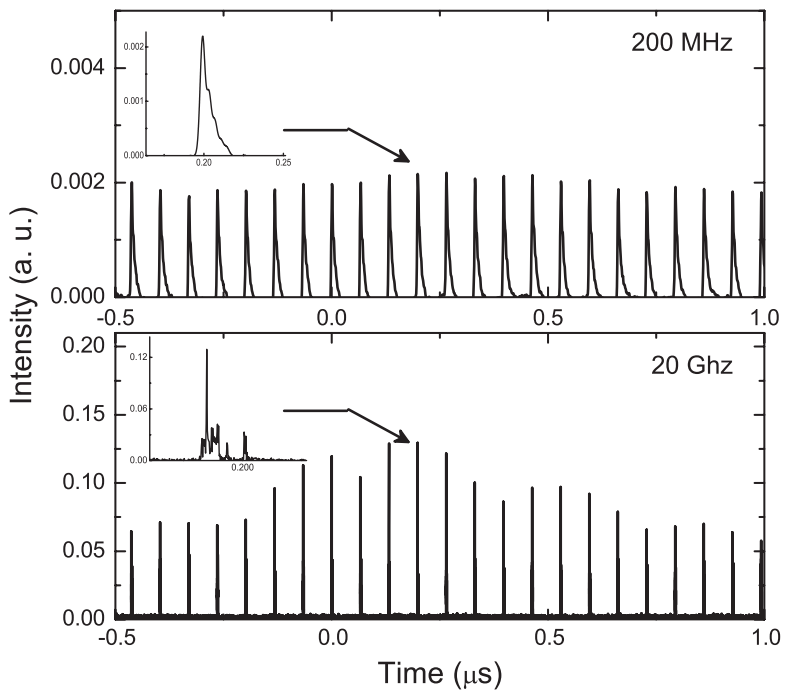

FIG. 3. Comparison between narrow [(a) $200 \mathrm{MHz}$ ] and wide [(b) $20 \mathrm{GHz}$ ] electronic bandwidth analysis of the same sequence of laser output pulse events. Insets: One pulse event is magnified in both cases. occurrence than in classical distributions at twice the SWH.

An important issue is the nature of these large amplitude fluctuations. To determine whether they result from internal pulse collisions rather than from the fluctuations of the total cavity energy, we analyze the same sequence of cavity output pulses with two largely different analyzing bandwidths. The narrow-bandwidth analysis $(200 \mathrm{MHz})$ does not resolve the internal bunch structure. In this case, the amplitude of the peak, as displayed on Fig. 3(a), mostly reflects the total bunch energy. We can see little variation of the amplitude: the bunch energy remains nearly constant from one roundtrip to the next. In contrast, the wide-bandwidth analysis $(20 \mathrm{GHz})$ clearly displays, in
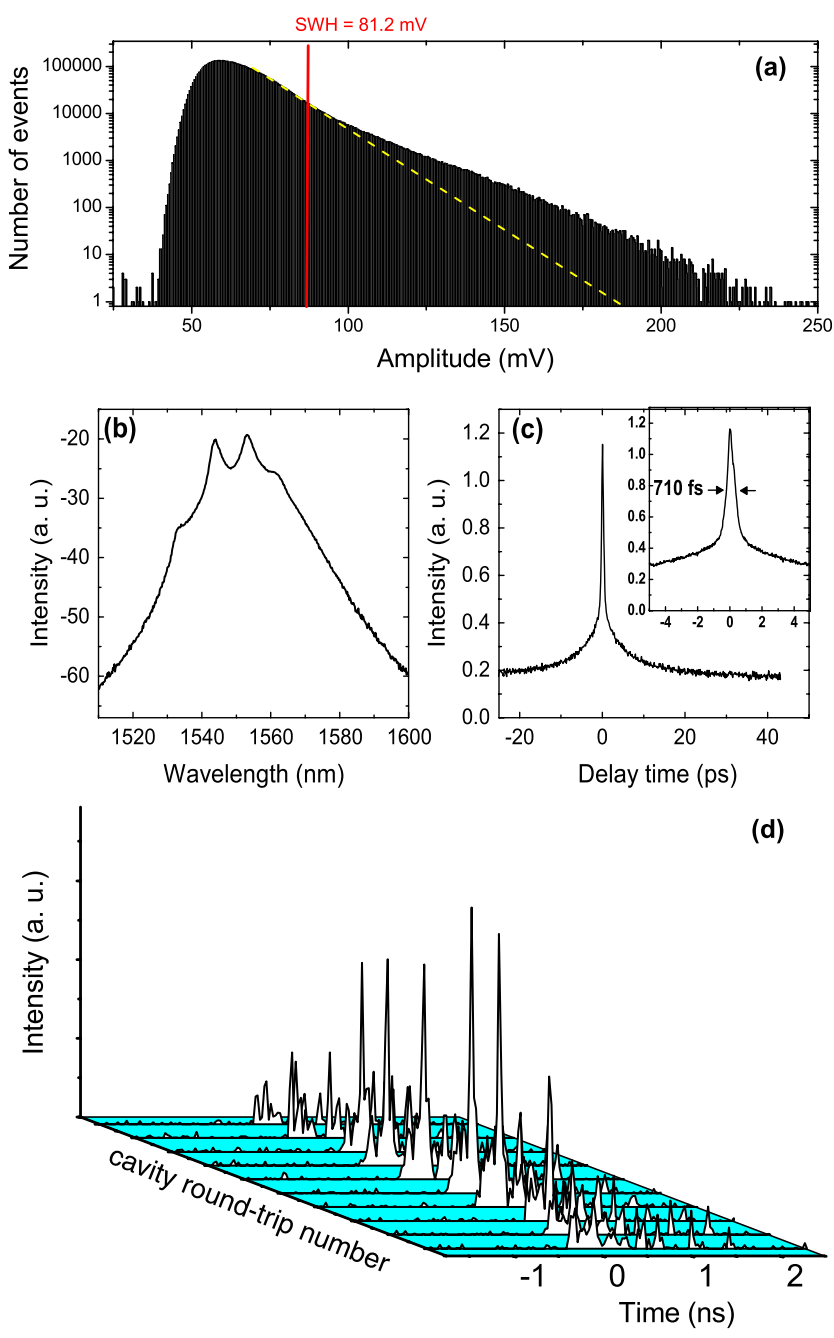

FIG. 4 (color online). (a) Histogram (log scale) showing a large deviation above the classical distribution (indicated by the yellow dotted line) for $6.8 \times 10^{6}$ trace events. RW events are observed at the level of up to 3 times the SWH. (b) Optical spectrum (log scale). (c) Optical autocorrelation trace, with a close-up view of the central coherence peak (inset). (d) Stroboscopic recording showing the intracavity evolution over successive roundtrips around a rogue wave event. 
Fig. 3(b), large amplitude fluctuations. We can conclude that the rogue waves presented here originate from the internal pulse interactions and collisions taking place within the bunch.

The degrees of freedom of the nonlinear cavity transfer function, which are activated by adjusting the orientations of the polarization controllers, allow us to optimize the occurrence of RW phenomena. Figure 4 presents a regime where significantly more RWs are detected. This is reflected by the large deviation above the classical distribution trend in Fig. 4(a), with RWs up to three times the SWH. The shape of the tail of the distribution agrees well with the one obtained in the numerical simulations presented in Fig. 11 of [17]. This regime is reached with a small variation of the cavity parameters used in Fig. 2, by tilting just one PC by 4 degrees. Consequently, the optical spectrum shown in Fig. 4(b) is similar to that in Fig. 2(b).

The optical autocorrelation trace is another important measurement, which is shown in Fig. 4(c). It is recorded at $4 \mathrm{~Hz}$, with $5 \times 10^{5}$ points, whereas the optical spectra have 2048 points recorded with an integration time of $1 \mathrm{~ms}$ for each point. The average duration of the basic single pulse constituent is estimated from the width of the central autocorrelation peak, which is 0.71 ps (inset of Fig. 4(c)). The large pedestal of the autocorrelation trace, which extends far beyond the 100-ps scanning range of the autocorrelator, can be attributed to the averaging of cross correlations between numerous pulses in ceaseless and rapid relative motions. Despite being observed in a narrow region in the multidimensional parameter space, the regime where RWs are observed is robust in the sense that stable averaged measurements, such as optical spectra and optical autocorrelation traces, can be repeatedly reproduced over hours. Interestingly, the two generic features put forward in [24] to explain rogue wave formation, namely granularity and inhomogeneity, are acting in our system. Firstly, the cavity field is fragmented into many pulses of a typical half-picosecond duration. Secondly, pulse bunching entails a notably inhomogeneous distribution of these temporal grains along the cavity.

Using successive recordings of oscilloscope traces, we also confirm the importance of the interactions between pulses in the multisoliton condensed phase, as observed earlier in numerical simulations [17]. A clear stroboscopic picture of the rogue wave dynamics over several cavity roundtrips is shown in Fig. 4(d).

In conclusion, we presented the first experimental demonstration of a new mechanism for rogue wave generation recently predicted by two independent theoretical works. Extreme peak-optical-intensity events result from the nonlinear interactions and collisions, in a strongly dissipative regime, of ultrashort optical pulses moving chaotically within the aggregated bunches. The distribution of intensity maxima, which is highly dependent on cavity parameters, satisfies the criteria of rogue waves within a certain region in the multidimensional parameter space. This observation is in good qualitative agreement with the theoretical predictions. Our results reveal the complex dynamics of nonlinearly interacting multiple pulses in a mode-locked laser cavity. They may attract the increasing attention of researchers in nonlinear optics, oceanography and other fields that may benefit from cross-fertilization of ideas.

C. L. and Ph. G. are supported by the Agence Nationale de la Recherche (project ANR-2010-BLANC-0417-01SOLICRISTAL) and the Région Bourgogne. J.M.S.C. acknowledges support from the Spanish Ministerio de Ciencia e Innovación under Contract No. FIS2009-09895. N. A. acknowledges the support of the Australian Research Council (Discovery Project DP110102068) and Volkswagen Stiftung.

[1] C. Kharif, E. Pelinovsky, and A. Slunyaev, Rogue Waves in the Ocean (Springer, Heidelberg, New York, 2009).

[2] A. R. Osborne, Nonlinear Ocean Waves and the Inverse Scattering Transform (Elsevier, Amsterdam, 2010).

[3] Special Issue on Rogue Waves-Toward a Unifying Concept, edited by N. Akhmediev and E. Pelinovsky [Eur. Phys. J. Special Topics 185, 1 (2010)].

[4] M. Shats, H. Punzmann, and H. Xia, Phys. Rev. Lett. 104, 104503 (2010).

[5] D. R. Solli, C. Ropers, P. Koonath, and B. Jalali, Nature (London) 450, 1054 (2007).

[6] H. Bailung, S. K. Sharma, and Y. Nakamura, Phys. Rev. Lett. 107, 255005 (2011).

[7] N. Akhmediev, J. M. Soto-Crespo, and A. Ankiewicz, Eur. Phys. J. Special Topics 185, 259 (2010).

[8] A. Demircan, Sh. Amiranashvili, C. Brée, Ch. Mahnke, F. Mitschke, and G. Steinmeyer, Phys. Rev. E (to be published).

[9] K. B. Dysthe and K. Trulsen, Phys. Scr. T82, 48 (1999).

[10] B. Kibler, J. Fatome, C. Finot, G. Millot, F. Dias, G. Genty, N. Akhmediev, and J. M. Dudley, Nature Phys. 6, 790 (2010).

[11] A. Montina, U. Bortolozzo, S. Residori, and F. T. Arecchi, Phys. Rev. Lett. 103, 173901 (2009).

[12] K. Hammani, C. Finot, J. M. Dudley, and G. Millot, Opt. Express 16, 16467 (2008).

[13] D. V. Churkin, O. A. Gorbunov, and S. V. Smirnov, Opt. Lett. 36, 3617 (2011).

[14] A. N. Pisarchik, R. Jaimes-Reátegui, R. Sevilla-Escoboza, G. Huerta-Cuellar, and M. Taki, Phys. Rev. Lett. 107, 274101 (2011).

[15] C. Bonatto, M. Feyereisen, S. Barland, M. Giudici, C. Masoller, J. R. Rios Leite, and J. R. Tredicce, Phys. Rev. Lett. 107, 053901 (2011).

[16] M. Kovalsky, A. Hnilo, and J. Tredicce, Opt. Lett. 36, 4449 (2011).

[17] J. M. Soto-Crespo, Ph. Grelu, and N. Akhmediev, Phys. Rev. E 84, 016604 (2011).

[18] A. Zaviyalov, O. Egorov, R. Iliew, and F. Lederer, Phys. Rev. A 85, 013828 (2012). 
[19] Ph. Grelu and N. Akhmediev, Nature Photon. 6, 84 (2012).

[20] S. Chouli and Ph. Grelu, Phys. Rev. A 81, 063829 (2010).

[21] L. M. Zhao and D. Y. Tang, Appl. Phys. B 83, 553 (2006).
[22] J.M. Soto-Crespo, M. Grapinet, Ph. Grelu, and N. Akhmediev, Phys. Rev. E 70, 066612 (2004).

[23] S. T. Cundiff, J. M. Soto-Crespo, and N. Akhmediev, Phys. Rev. Lett. 88, 073903 (2002).

[24] F. T. Arecchi, U. Bortolozzo, A. Montina, and S. Residori, Phys. Rev. Lett. 106, 153901 (2011). 